\title{
Review
}

\section{Ageing, autoimmunity and arthritis Perturbations of TCR signal transduction pathways with ageing - a biochemical paradigm for the ageing immune system}

\author{
Tamàs Fülöp Jr ${ }^{1,2,3}$, Anis Larbi ${ }^{1,2}$, Gilles Dupuis ${ }^{2,4}$ and Graham Pawelec ${ }^{5}$
}

\author{
${ }^{1}$ Research Center on Ageing, Sherbrooke Geriatric University Institute, University of Sherbrooke, Quebec, Canada \\ ${ }^{2}$ Graduate Program in Immunology, University of Sherbrooke, Quebec, Canada \\ ${ }^{3}$ Geriatric Service, University of Sherbrooke, Quebec, Canada and Geriatric Division, University of Franche-Comté, Faculty of Medicine, Hôpital \\ Jean Minjoz, Besançon, France \\ ${ }^{4}$ Department of Biochemistry, Faculty of Medicine of Sherbrooke, University of Sherbrooke, Quebec, Canada \\ ${ }^{5}$ Tübingen Ageing and Tumour Immunology Group, Section for Transplantation Immunology and Immunohaematology, Second Department of \\ Internal Medicine, University of Tubingen Medical School, Zentrum für Medizinsche Forschung, Tubingen, Germany \\ Correspondence: Tamàs Fülöp Jr (e-mail: Tamas.Fulop@USherbrooke.ca)
}

Received: 28 Jul 2003 Revisions requested: 22 Aug 2003 Revisions received: 1 Oct 2003 Accepted: 13 Oct 2003 Published: 16 Oct 2003

Arthritis Res Ther 2003, 5:290-302 (DOI 10.1186/ar1019)

(c) 2003 BioMed Central Ltd (Print ISSN 1478-6354; Online ISSN 1478-6362)

\begin{abstract}
It is widely accepted that cell-mediated immune functions decline with age, rendering an individual more susceptible to infection and possibly cancer, as well as to age-associated autoimmune diseases. The exact causes of T-cell functional decline are not known. One possible cause could be the development of defects in the transduction of mitogenic signals following TCR stimulation. This T-cell hyporesponsiveness due to defects of signalling through the TCR either from healthy elderly subjects or from individuals with autoimmune diseases such as rheumatoid arthritis or systemic lupus erythematosus results in an impaired ability to mount efficient immune responses and to maintain responsiveness to foreign antigens. This implies that a high proportion of autoreactive T cells might accumulate either intrathymically or in the periphery. T-cell anergy and differential TCR signalling could thus also be key players in the disruption of tolerance and the onset of autoimmune diseases. The increasing number of the elderly may lead to an increase of clinically important autoimmune diseases. We will review the signal transduction changes through the TCR-CD3 complex in T lymphocytes from healthy elderly subjects, which result in a modification of the activation of transcription factors involved in IL-2 gene expression leading to decreased IL-2 production. The putative contribution of altered T-cell signalling with ageing in the development of autoimmune diseases will be also discussed.
\end{abstract}

Keywords: ageing, arthritis, autoimmunity, lipid rafts, T-cell receptor signalling

\section{Introduction}

It is widely accepted that cell-mediated immune functions (cytotoxicity, delayed-type hypersensitivity, etc.) decline with age $[1,2]$. These age-associated immunological changes render an individual more susceptible to infection and possibly cancer, as well as to age-associated autoimmune disorders; they may also contribute to atherosclerosis and Alzheimer's disease [3-5]. There is still no clear consensus as to why cell-mediated immunity declines with age. It is generally believed that age-related immune deficiency develops coincident with the gradual involution of the thymus gland and, consequently, that thymic-related (T-cell) functions are the most profoundly affected. It has recently been suggested that the alterations observed with ageing are a reflection of an accumulation of relatively inert memory $T$ cells and a consequent

APC = antigen-presenting cell; DRM = detergent-resistant microdomain; ERK = extracellular signal-regulated kinase; IFN = interferon; IL = interleukin; ITAM = immunoreceptor tyrosine-based activation motif; LAT = linker of activated T cells; mAb = monoclonal antibody; MAPK = mitogenactivated protein kinase; $\mathrm{MHC}=$ major histocompatibility complex; NF = nuclear factor; NFAT $=$ nuclear factor of activated $\mathrm{T}$ cells; PKC $=$ protein kinase C; pLAT, tyrosine-phosphorylated LAT; PTK = protein tyrosine kinase; RA = rheumatoid arthritis; SLE = systemic lupus erythematosus; $\mathrm{TCR}=\mathrm{T}$-cell receptor; $\mathrm{Th}$ cells $=\mathrm{T}$ helper cells; $\mathrm{ZAP}=$ zeta-associated protein 
reduction of reactive naïve T cells [6,7]. Nevertheless, none of these findings alone can explain satisfactorily the decline of cell-mediated immunity with age.

The proliferation and clonal expansion of Tlymphocytes is mostly controlled by interactions between the cytokine IL-2 and its cellular receptor [8] following TCR ligation [9]. It is now well accepted that the induction of $\mathrm{IL}-2$ secretion decreases with age in mice, in rats and in humans [10]. It seems that such defects occur in the transduction of mitogenic signals following TCR stimulation. Indeed, recent studies suggest that alterations in, for example, tyrosine kinase activity, intracellular free calcium, inositol phosphates, protein kinase $\mathrm{C}$ (PKC), and so on, may all contribute to changes in signal transduction with ageing [11-15]. Although the signalling machinery in T cells is extremely complicated and many steps remain to be clarified, the agerelated change in T-cell signal transduction may be one of the most important causes of cell-mediated immune response decline with ageing. Thus, considering the decrease of cell-mediated immune response with ageing and the possibility that alterations in signal transduction may be one of the causes, we will review the signal transduction changes in Tlymphocytes from healthy elderly subjects that would translate into a modification of the activation of transcription factors involved in IL-2 gene expression leading to decreased IL-2 production. The putative contribution of the altered T-cell signalling with ageing in the development of autoimmune diseases will also be discussed.

\section{TCR and costimulatory signalling pathways}

First, we will briefly summarize the very complex signalling events occurring during TCR-mediated T-cell activation, which leads to a perfectly coordinated immune response and is essential for understanding the age-related changes that may later lead to deregulated immune responses. Engagement of the TCR by antigen in the context of the MHC [16] interacting with the clonotypic portion of the TCR-CD3 complex as well as the CD4 or CD8 subunits results in a rapid early cascade of intracellular signalling events. This cascade leads to activation of cytoplasmic and nuclear factors that are necessary, but not sufficient, for IL-2 gene transcription. This is referred to collectively as signal I [17]. Additionally, CD28 and other less well-investigated costimulatory pathways must be activated to ensure a complete and efficacious T-cell activation, collectively designated signal II [18,19].

Once the TCR is effectively engaged, cytoplasmic protein kinases (PTKs) are recruited, which have been demonstrated to be tightly associated with different components of the CD3 complex and other T-cell antigens (CD4, CD8). The activation of the src-like cytoplasmic tyrosine kinases (Ick, fyn) leads to phosphorylation of a number of immunoreceptor tyrosine-based activation motif (ITAM) containing proteins including TCR $\zeta$ [20], and this promotes the recruit- ment of a syk family member protein kinase, zeta-associated protein (ZAP)-70, and induces its activation [21]. Lck and Fyn functions are also regulated by the tyrosine phosphatase CD45, as well as by some recently identified phosphatase complexes [22]. Activated ZAP-70 phosphorylates the linker of activated T cells (LAT), one of the most important T-cell adapter proteins [23]. LAT then becomes a scaffold protein for the recruitment of multiple partners including the adaptor proteins Gads and Grb2 and the enzymes of phospholipid metabolism phosphatidylinositol-3 kinase and PLC $\gamma 1$ [24]. Whereas phosphatidylinositol-3 kinase creates novel binding sites in the inner leaflet of the plasma membrane for the recruitment of pleckstrin homology domaincontaining proteins, activated PLC $\gamma 1$ generates InsP3, which is responsible for the mobilization of $\mathrm{Ca}^{2+}$ [25].

LAT-mediated recruitment of the Grb2-Sos complex to the membrane initiates Ras activation. The GTP-bound p21 ras interacts with the serine/threonine kinase Raf-1, leading to the activation of mitogen-activated protein kinases (MAPKs). There are actually three MAPK subfamilies: extracellular signal-regulated kinases (ERK1 and ERK2), p38 and JN kinases. The downstream signalling pathways mediated by MAPKs are considered essential for normal cell growth and proliferation [26]. PKC activation contributes to the activation of MAPK, most probably via c-Raf-1 activation. Activated MAPKs can phosphorylate, at least in vitro, a variety of transcription factors including c-jun, c-myc, $\mathrm{c}$-fos and p62TCF (Elk-1). The rise in intracellular calcium activates the calcium/calmodulin-dependent serine/threonine kinases and phosphatases, such as calcineurin, which allows the cytoplasmic component of nuclear factors to be activated and to move to the nucleus [27]. For instance, NF-ATp will combine with newly formed Fra-1 (a member of the fos family) and JunB proteins (induced by the PKC pathway) to create the nuclear factor of activated $T$ cell (NFAT) complex. Finally, it has been shown that TCR-mediated signal transduction causes the dissociation of the NF$\kappa B$ transcription factor from the inhibitory factor $\mathrm{kBB}$, probably via PKC $\theta$-dependent phosphorylation of IkB [28].

LAT-associated Gads bring SLP-76 to the plasma membrane where it becomes phosphorylated, allowing its interactions with the exchange factor Vav, the adaptor proteins Nck and SLAP-130/Fyb, and the tec PTK Itk. Vav and SLAP-130/Fyb provide a link between T-cell activation, upregulation of integrin affinity/avidity as a result of insideout signalling, and reorganization of the cell cytoskeleton [29]. The TCR ligation-initiated complex and the interactive signal transduction pathways thus lead to T-cell activation, resulting in differentiation, IL-2 production, clonal expansion, survival and effectors functions. Although the engagement of the TCR provides an essential signal to $T$ cells, commitment to proliferation, to differentiation and to survival will not occur unless a secondary signal is provided by ligation of, for example, CD28 [30,31]. 
Although the pathways are still not fully established, signalling through CD28 is associated with phosphatidylinositol-3 kinase that may involve activation of $\mathrm{AkT} / \mathrm{PkB}$ and other kinases. Phosphatidylinositol-3 kinase could be a potent activator of the $\mathrm{Ca}^{2+}$-independent $\mathrm{PKC}$, and its isoforms. Regardless, CD28 costimulation is essential for JN kinase and NF- $\mathrm{KB}$ activation, which are necessary for activation of the IL-2 promoter [30]. CD28 thus contributes to the overall increase of the efficacy of TCR signalling at multiple levels. Moreover, it has recently become evident that CD28 assists in the assembly of the cytoskeleton and the recruitment of lipid rafts to the TCR immunological synapse. CD28 thus controls a wide range of events in naive $\mathrm{CD} 4^{+} \mathrm{T}$ cells, including a decrease of the TCR signalling threshold. In contrast, the major effect of CD28 in $\mathrm{CD}^{+}$memory T cells is to enhance the TCR response, whereas its role in $\mathrm{CD}^{+} \mathrm{T}$ cells is less clearly defined. This two-signal concept has particular relevance for understanding T-cell tolerance and age-associated changes.

Much progress has very recently been made in our comprehension of how all these different events and pathways are spatially linked to form an optimal signalling complex. The notion of the immune synapse has emerged and is now widely accepted. This is an informational synapse that relays information across a quasi-stable cell-cell junction during TCR interactions with the MHC-peptide complex [32]. A redistribution of the signalling components takes place in two major compartments: central supramolecular activation clusters enriched in TCR and CD28, and peripheral supramolecular activation clusters containing leukocyte functional antigen-1 and talin. Another major advance in our understanding is the recognition of the existence of special membrane domains called rafts, small regions of detergent-resistant complexes in the membrane [33] enriched in glycosphingolipids and cholesterol, defined as a liquid-ordered phase in the membrane. Lck and LAT are targeted with other signalling molecules, including members of the Src PTK family, heterotrimeric G proteins and Ras, to glycolipid-enriched microdomains as a consequence of post-translational palmitoylation [34]. After TCR ligation, phosphorylated TCR, ZAP-70, Shc and PLC also localize to these microdomains. The inducible assembly of signalling complexes within these microdomains is a prerequisite for efficient TCR signal transduction [35]. CD28, CD2, CD5 and lymphocyte function-associated antigen-1 play a major role in recruiting lipid rafts to the TCR synapse. It is of note that recent studies indicate that Lck and LAT are present in different lipid rafts [36]. The combination of these signalling events results in the formation/activation of transcription factors.

The formation of the TCR synapse and the supramolecular activation clusters provide a stable arrangement through which the TCR can establish an optimal threshold for an optimal number and avidity of TCR-MHC peptide interactions [16]. The cumulative effect of integrated signalling events is to deliver a high fidelity signal that leads to T-cell activation manifesting as proliferation, differentiation, apoptosis, anergy, and/or development of memory or effector functions, all modulated by the relative proportions of T-cell subpopulations, costimulatory receptors, membrane composition, type of antigen-presenting cell (APC) or cytokine balance. When there is a disruption in this fully integrated signalling cascade by changes occurring either physiologically (including ageing) or pathologically (including cancer and autoimmune disease), this leads to an altered immune response.

\section{TCR and costimulatory signalling changes with ageing: a biochemical paradigm for the ageing immune system}

It is well accepted that the early signalling events during stimulation via the TCR-CD3 complex are altered with ageing in Tlymphocytes [11,14], as is the subsequent expression of early-activation surface markers such as CD69 and CD71 [37]. These changes might arise from decreased numbers of TCR per cell or changed TCR reexpression after stimulation, from alterations to the cell membrane, from changes in the TCR signalling pathways or from alterations of coreceptors.

\section{Receptor number}

A question that is still not completely settled is whether the TCR receptor number does change with ageing. This could be due to a decrease of expression or re-expression of TCR. In T lymphocytes, experimental data show various changes in TCR receptor number, but the bulk of the experimental evidence suggests that with normal ageing the TCR number does not change significantly [38]. However, TCR re-expression could be altered as a consequence of the alteration in CD28 and its signalling. Indeed, experimental data clearly show that the expression of CD28 is decreasing with ageing [39].

\section{Plasma membrane and lipid rafts of $T$ cells}

Specificity and fidelity of signal transduction are crucial for cells to respond efficiently to changes in their environment. This is achieved in part by the differential localization of proteins that participate in signalling pathways. The lipid bilayer of the plasma membrane is organized into cholesterol and glycosphingolipid-rich microdomains, also called rafts $[40,41]$. It was recently shown that TCR ligation induces a redistribution of tyrosine-phosphorylated proteins into lipid rafts. Experimental data collectively indicate that the plasma membrane, via its special organization in lipid rafts, plays an important role in signal transduction via the TCR [35]. It has been known for some time, however, that there is an alteration in the physicochemical status of the plasma membrane, leading to increased rigidity and decreased fluidity with ageing [42]. This may well affect raft formation [43]. 
What is our actual knowledge concerning changes to these immune synapses and lipid rafts with ageing? Very limited information exists concerning this issue in the context of TCR signalling. Miller and colleagues $[44,45]$ recently demonstrated an alteration in several components of this signalling complex with ageing in memory $T$ cells [44] as well as in naïve T cells [45]. The most important findings concern the reduced activation of several raftassociated or recruited proteins, such as LAT, PKC, and Vav in T cells of aged mice. There was an age-associated decline in the proportion of $\mathrm{CD} 4^{+} \mathrm{T}$ cells that redistributed LAT and Vav to the T cell-APC synapse upon ligation of the TCR. Similarly, but at the level of the nucleus, the transcription factor NF-ATc had a decreased redistribution upon stimulation. Most probably the decreased recruitment of the signalling molecules to the synapses is responsible for decreased NF-ATc translocation to the nucleus, playing a role in decreased IL-2 gene expression [46]. The reasons for this altered tyrosine phosphorylationmediated activation are not yet known. Together, these data show that alterations in the immune synapses and lipid rafts can be found in both naive cells and memory cells with ageing. How all these affect the distinct functioning of the cells will be the target of future research.

We ourselves were also interested to investigate in human T cells whether the alteration of the cholesterol content of the cell membrane, and in consequence that of lipid rafts, could modulate the activation of various signalling pathways and functions [47]. In agreement with previous findings, it was confirmed that the cholesterol content of the T-cell plasma membrane was significantly increased with ageing, which could explain its increasing rigidity with age [15]. We recently found that the higher levels of cholesterol were contained in lipid rafts (fractions 1-3) of T-cell lysates that were more than twofold higher in the case of elderly donors as compared with young donors (unpublished data). Higher concentrations of cholesterol in heavy sucrose density fractions (fractions 4-9) were also observed. Why the levels of cholesterol are increased in detergent-resistant microdomains (DRMs) from elderly normolipidaemic individuals is not known. These results may suggest, however, that the cellular regulation of cholesterol metabolism is altered with ageing and could either involve abnormal regulation of cellular cholesterol export or altered production intracellularly $[43,48]$.

Ageing has been reported to be associated with a decrease in the fluidity of the T-cell plasma membrane [49]. This alteration in physicochemical properties could affect the formation of DRMs and their lateral mobility as a result of the high levels of plasma membrane cholesterol. Indeed, T cells exposed to anti-CD3 or a combination of anti-CD3 and anti-CD28 mAb induced significantly decreased raft coalescence in T cells of elderly subjects independent of the stimuli used. The observed decrease in
DRM movement in the membrane bilayer may contribute to the defects in T-cell proliferation seen with ageing [50] as plasma membrane fluidity is needed for optimal T-cell interactions with APCs [51]. The combination of anti-CD3 and anti-CD28 mAb was twice as effective as the anti$\mathrm{CD} 3 \mathrm{mAb}$ in triggering the coalescence of fluorescence. These observations are in agreement with the findings of Viola and colleagues [52], who showed that simultaneous cross-linking of the TCR complex and CD28 was needed to induce coalescence of lipid rafts in human T cells.

TCR ligation also induces a redistribution of tyrosinephosphorylated proteins into lipid rafts that include the TCR components, phospholipase C, GRB2, ZAP-70 and Lck [53]. A decline in the levels of tyrosine-phosphorylated proteins following the activation of $T$ cells from aged humans [12] or from mice [54] has been reported and these differences could be reflected in a differential distribution in DRMs [55]. Our data show that the activation of T cells resulted in an increase of pLck levels in DRMs of young subjects, but not of elderly subjects.

LAT is an essential component of the assembly machinery of signal transduction in T lymphocytes [56]. Activation of Tlymphocytes from young and old subjects results in marked differences in DRM recruitment of LAT. Although the bulk of the protein remained associated with the heavy fractions, its relative distribution was more than fourfold increased in DRMs of young individuals. This was not the case in DRM fractions of T-cell lysates from elderly subjects. Analyses of the tyrosine-phosphorylated LAT (pLAT) distribution in DRMs showed $>10$-fold increases in DRMassociated pLAT in lysates of activated T cells from young subjects (unpublished data). However, the relative increase in the recruitment of pLAT to DRMs was much lower in T-cell lysates from elderly individuals. These findings are in agreement with those observed in the case of T cells from aged mice [45], and they may explain the defects in downstream pathways of T-cell activation with ageing and the chronic inflammatory process associated with ageing [57]. These data reveal significant alterations in the properties of DRMs with ageing, which include an increase in cholesterol content, impaired DRM coalescence and selective differences in the recruitment of key proteins involved in T-cell signalling (see Fig. 1).

We have explored the effects of a cholesterol-extracting molecule, methyl $\beta$-cyclodextrin, which is known to disrupt rafts in T cells and, as a consequence, to alter signal transduction upon TCR ligation [58]. Unlike other cholesterolbinding agents that incorporate into membranes, methyl- $\beta$-cyclodextrin is strictly surface-acting and selectively extracts plasma membrane cholesterol [58]. It was found that the effect of methyl- $\beta$-cyclodextrin was quite different on the cholesterol content and signalling molecules of young subjects' T lymphocytes versus elderly subjects' 


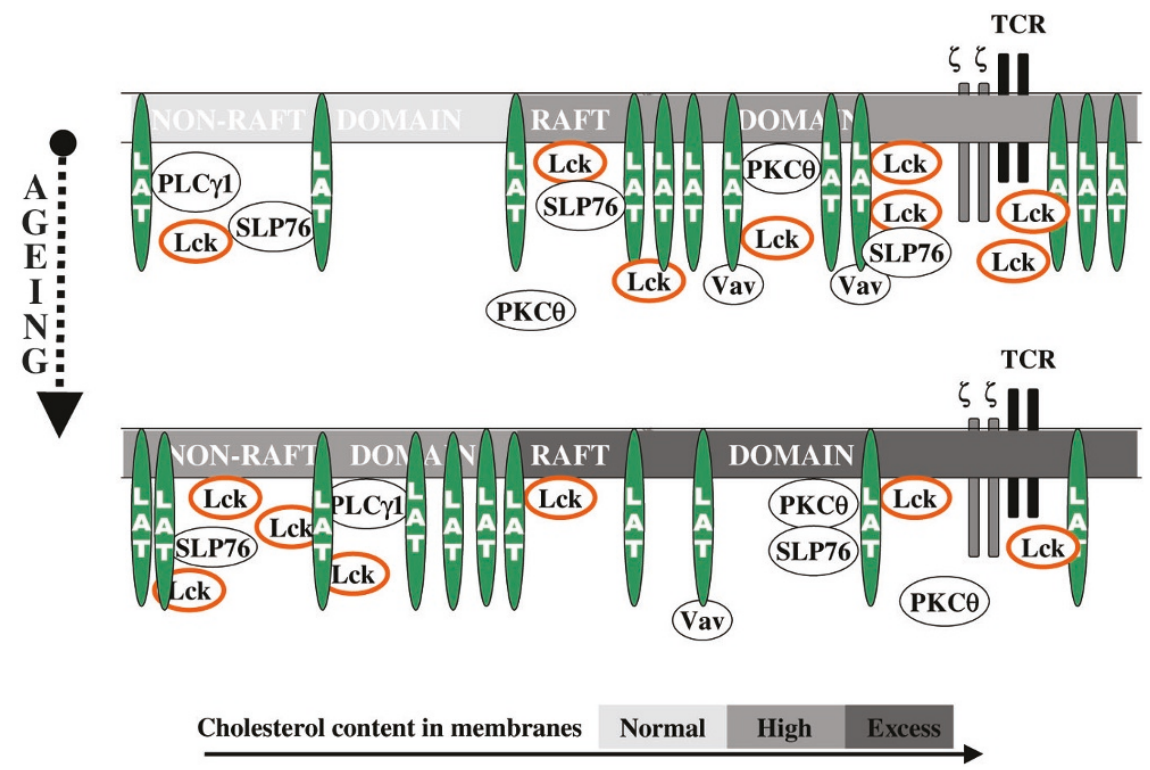

Schematic conceptualization of the changes with ageing in lipid raft composition following plasma membrane changes in cholesterol content and their effects on early signalling events. LAT, linker of activated T cells; PKC, protein kinase C; TCR, T-cell receptor.

T lymphocytes. Extraction of cholesterol in T cells of young healthy subjects had a very dramatic signal-disrupting effect, while in T cells of healthy elderly subjects Lck, ERK1 and ERK2 phosphorylations were enhanced compared with the nontreated T cells. Proliferation of these cells was also improved, although without attaining the level of young subjects. These data further support an alteration at the level of the lipid rafts and the idea that membrane cholesterol plays a critical role in the homeostatic regulation of signalling pathways in T cells [43,48], which is altered with ageing. The manipulation of cholesterol content might thus have an immunomodulating effect. There is still much to be learned in relation to this new concept of immune signal transduction during TCR ligation with ageing in different T-cell subpopulations.

In addition to the increase in cholesterol content, alterations to the membranes of cells associated with ageing could be due to oxidation by free radicals [59]. Oxidative stress has been shown to damage cell membranes, altering in vitro binding activity of AP-1 (activating protein-1) and suppressing in vitro concanavalin A-induced T-cell proliferation and IL-2 production [60].

\section{Several age-dependent signalling pathways show alterations upon TCR ligation}

As already mentioned, the first step in TCR-mediated signal transduction is the activation of different tyrosine kinases, leading to the tyrosine phosphorylation of several were found altered with ageing either in experimental animals or in humans. The level of tyrosine phosphorylation of p59fyn and ZAP-70 kinases is impaired in T cells from old mice activated through the TCR-CD3 complex. In human T cells, an age-related defect is observed in tyrosine-specific protein phosphorylation after activation via TCR-CD3 complexes, CD4 and IL-2 receptors [63]. In addition, a reduction in p59fyn activity was found in some elderly subjects without compensation by p56lck activity. We have also recently shown a substantial decrease in p56lck activity in T lymphocytes of healthy elderly subjects [15]. Consequently, as in mice, ZAP-70 activity is also decreased in T cells. Not unexpectedly, there are some discrepancies in the protein tyrosine kinase activity measurement with ageing, but together these results suggest that the activation and functions of the early signalling protein tyrosine kinases induced by TCR ligation are altered with ageing.

It is now well documented that other early events related to protein tyrosine phosphorylation following TCR activation are altered with ageing, such as the generation of myo-inositol 1,4,5-trisphosphate, intracellular free calcium mobilization and PKC translocation [11]. Other studies, as well as our own, concerning the changes in cytoplasmic free calcium ion concentration as an index of the very early events in the T-cell activation process, have shown that the anti-CD3 mAb-induced mobilization of cytoplasmic free $\mathrm{Ca}^{2+}$ declines with age $[64,65]$. There are some studies concerning PKC activity with ageing in immune 
cells. Proust and colleagues [65] have shown defects in translocation of PKC in Tlymphocytes of old mice. Our own studies also demonstrated an altered PKC isozyme distribution and translocation in human T lymphocytes with ageing under anti-CD3 mAb stimulation. These alterations in PKC activation might markedly contribute to the observed impairment of T-cell activation with ageing. Moreover, the results suggest that an inability to elevate PKC activity after TCR stimulation may originate from alterations in the early signal transduction events.

Only few data exist concerning phosphatase activity in $T$ cells with ageing. There is increasing experimental evidence that the balance between tyrosine kinases and phosphatases is essential for the maintenance of the resting status and for activation [17]. CD45 is a receptorlike protein tyrosine phosphatase expressed on all nucleated haematopoietic cells. One key function of CD45 is to serve as a positive regulator of src tyrosine kinases, by opposing Csk function and by dephosphorylating the negative regulatory $\mathrm{C}$-terminal tyrosine of src tyrosine kinases. CD45-protein tyrosine phosphatase activity in old cells after CD3 stimulation is not changed compared with that in young cells [66]. No data exist thus far on changes of activities of the other phosphatases with ageing. It can be supposed, however, that the interaction between Cbp/PAG (csk-binding protein/phosphoprotein associated with glycosphingolipid-enriched microdomain) and Csk may be altered, and therefore the release of Csk could also be altered. Further studies are needed in this specialized field. These data together suggest that early events in human T-cell activation are altered with ageing (see Table 1).

Data are starting to accumulate showing that events more distal from tyrosine kinases are also altered with ageing [66]. Data indicate that the Ras-MAPK/ERK pathways are also changed with ageing [67]. Whisler and colleagues [68] have shown that $50 \%$ of old subjects had a reduction in MAPK activation. ERK2 activation was correlated with the ability of T cells to produce IL-2 and to proliferate. Diminished ERK2 activation may thus represent one of the rate-limiting steps for IL-2 production by T cells of old individuals. Furthermore, we have recently shown that there is an alteration with ageing in MAPK/ERK as well as in p38 activation in T cells of the elderly compared with young subjects following TCR stimulation [50], as already demonstrated [69]. These data altogether suggest that an alteration exits in the Ras-MAPK signalling pathway with ageing, leading to decreased T-cell functions.

\section{Transcription factor changes with ageing}

The transcription factors studied to date show age-related decreased activity. Data on the effect of age on the NFAT complex show an age-related decline in NFAT binding to nuclear extracts of T lymphocytes from rats [68]. The age-
Table 1

\begin{tabular}{|c|c|}
\hline Signalling alteration & Role in T cells \\
\hline Intracellular free $\mathrm{Ca}^{2+}$ & Early T-cell activation \\
\hline $\begin{array}{l}\text { Myo-inositol } \\
\text { 1,4,5-trisphosphate production }\end{array}$ & Calcium release \\
\hline $\begin{array}{l}\text { Protein kinase } \mathrm{C} \text { isoform } \\
\text { translocation }\end{array}$ & T-cell activation \\
\hline CD69 expression & Activation surface marker \\
\hline CD71 expression & Activation surface marker \\
\hline CD28 expression & Costimulatory molecule \\
\hline Membrane fluidity & Protein trafficking \\
\hline Cholesterol content & Lipid rafts movement \\
\hline Rafts-associated proteins & $\begin{array}{l}\text { Early T-cell activation, immune } \\
\text { synapse formation }\end{array}$ \\
\hline NF-AT distribution & Calcium signalling \\
\hline $\begin{array}{l}\text { Regulation of cellular } \\
\text { cholesterol }\end{array}$ & Molecule sorting, lipid rafts formation \\
\hline Rafts coalescence & $\begin{array}{l}\text { T-cell activation, immune synapse } \\
\text { formation }\end{array}$ \\
\hline Lck activation & ZAP-70, ITAM phosphorylation \\
\hline $\begin{array}{l}\text { Linker of activated T cells } \\
\text { activation }\end{array}$ & Signalling platform \\
\hline Fyn activation & ZAP-70, ITAM phosphorylation \\
\hline ZAP-70 activation & Linker of activated T cells activation \\
\hline $\begin{array}{l}\text { Extracellular signal- } \\
\text { regulated kinase activation }\end{array}$ & Late T-cell activation, proliferation \\
\hline p38 activation & Late T-cell activation, proliferation \\
\hline Proteasome activity & NF- $\kappa B$ relocalization \\
\hline
\end{tabular}

related decline observed in NFAT binding activity could arise through changes in its cytoplasmic component (i.e. NFAT-p). A decrease in calcium signal generation with age, through calcineurin, could then contribute to the decreased NFAT binding activity as observed in nuclear extracts isolated from splenocytes of old rats.

The other important transcription factor for IL-2 secretion is NF- $\kappa \mathrm{B}$. This protein is constitutively expressed and remains in the cytoplasm, bound to an inhibitory protein IkB, prior to activation. When stimulated, T cells generate reactive oxygen species, changing the redox status and leading to phosphorylation and ubiquitination of IkB. This results in dissociation of IkB from NF-KB followed by the degradation of $\mathrm{lkB}$ by the proteasome. This in turn results in the translocation of active NF- $\mathrm{KB}$ to the nucleus. Studies on NF-KB showed a decrease in its activation in mice and in humans mostly due to a decreased inactiva- 
tion of IkB by the proteasomes [70]. Decreased proteasome activity with ageing was also reported in tissues of old rats and in cultured human fibroblasts undergoing replicative senescence. The alteration of proteasome activity with ageing was attributed to oxidative stress.

Based on these results, we conclude that ageing does influence the activation of transcription factors following T-cell stimulation, which may result in decreased IL-2 production (see Table 1). However, more studies are needed to elucidate the complete mechanism of this decline under different experimental circumstances.

\section{T-cell subpopulation changes with ageing}

It is well known that the priming status of Tlymphocytes determines their response to stimulation as well as their ultimate function. In this context the distinction between naiive cells and memory cells becomes essential. Furthermore, important recent findings indicate that there is a difference between naïve and memory cells and effector cells in terms of lipid raft distribution and protein content [71]. Naïve T cells have fewer rafts in their plasma membrane and require CD28 costimulation to amplify TCR signalling by recruiting rafts to the TCR-ligand contact site. By contrast, effector and/or memory T cells have more rafts in their plasma membrane; amplification of signalling is thus able to occur in the absence of CD28 costimulation.

It is generally quite well accepted that the number of $T$ cells does not change markedly with ageing. In contrast, there is a consensus that ageing is accompanied by changes in the proportions of T-cell subpopulations. There is a higher number of T cells with the CD45RO' 'memory' phenotype and much less with the CD45RA' 'naïve' phenotype in peripheral blood mononuclear cells, although this is of course an oversimplification, albeit a useful one [7]. No matter which marker we use the numbers of naïve cells dramatically decreases with ageing, mainly among $\mathrm{CD}^{+}$cells. These alterations lead to decreased proliferative responses and to a decreased response to new antigens, but possibly to a better response to antigens already encountered.

CD28 may be considered a biomarker of ageing in T cells. The proportion of CD28 ${ }^{+}$T cells decreases in vivo with ageing and in in vitro culture models [72,73]. Effros showed a decreased percentage of Tlymphocytes that are $\mathrm{CD}_{28}{ }^{+}$in the CD8 T-cell subpopulation [7]. In addition, the average telomere lengths in the CD28- T cells are decreased, indicating that these cells have undergone numerous cell divisions. This type of proliferative senescence might be responsible for the accumulation of oligoclonal CD28- populations in elderly subjects. Very few data exist relating these changes in T-cell subpopulations to the signal transduction changes observed in peripheral signal transduction changes demonstrated in the whole population of T cells with ageing are also observed in isolated naïve T cells [45]. However, several findings indicate that the alterations rather reflect the behaviour of the accumulated memory T cells with ageing. More in-depth analyses are needed to establish the exact contribution of the observed signal transduction changes, in the various T-cell subpopulations, with ageing.

Other recently described Tregulatory cells, such as $\mathrm{CD}^{+} / \mathrm{CD}^{2} 5^{+}$and natural killer T-cell subsets, could also play an important role in ageing and in autoimmune diseases such as diabetes mellitus type 1 [74]. It was shown that these peripheral T-cell subsets actively contribute to the maintenance of self-tolerance. What role these T-cell subsets play in ageing is actually unknown. Future research will certainly bring further information on these cells, in relation to ageing, and help to integrate them into the complicated network of T-cell subpopulations.

\section{Putative contribution of TCR signalling changes in ageing to the increase of autoimmune disorders}

The question arises as to why the risk of several diseases having an autoimmune pathogenesis increases with age. In fact, even though some autoimmune diseases occur in childhood, others are typically related to ageing and often develop after 50 years of age, such as rheumatoid arthritis (RA) [75]. It is also of note that many alterations resembling those found in autoimmune diseases, especially in RA and in systemic lupus erythematosus (SLE) [19], exist in healthy aged individuals, including an increase in autoantibodies, a contraction in diversity of naïve T cells with increased oligoclonality, an increase in memory CD8 ${ }^{+}$CD28- $T$ cells, telomere shortening in T cells and T-cell signal transduction alterations - albeit, however, often without the appearance of overt autoimmune disease [2].

Many aspects of the ageing immune system predispose to the development of autoimmune diseases. Thymic involution has been evoked to be fundamental as this causes a drastic decrease in naïve $T$ cells and consequently leads to the contraction in diversity of the T-cell repertoire. During adulthood, the size of the peripheral T-cell pool is maintained, consequent to the homeostatic proliferation of peripheral T cells that also influences the T-cell repertoire [76]. In fact, self-replication of naïve T cells, driven by selfantigens, is accelerated to help compensate for reduced thymic output, and this could ultimately lead to clonal expansion of cells with much higher affinity for self at the expense of those that are weakly self-reactive but specific for nominal antigen [75-77]. This process contributes to physiological homeostasis by filling the unoccupied space with phenotypically naïve cells lacking effector functions [78]. In RA, however, these self-reactive T cells may 
acquire properties with potential for tissue injury resembling those of certain senescent cells in the course of ageing [79]. When lymphopaenia was induced in RA patients aged older than 40 years, a similar phenomenon was observed: the refilling of the space caused by decreased thymic output of naïve T cells and the repertoire of peripheral $T$ cells ultimately filling the space was severely contracted [80]. These peripheral naïve T cells underwent considerable proliferative activity indicated by reduced telomere length.

Homeostatic proliferation is dependent on TCR triggering. The two-signal model of T-cell activation has led to the notion of T-cell anergy as a mechanism of maintenance of tolerance in the periphery [18]. T-cell anergy was originally described as the result of a lack of costimulation during initial TCR engagement with antigen [19]. Although such T-cell tolerance may represent a mechanism for peripheral tolerance, it may also play a role in the development of autoimmune diseases [81]. Indeed, in the absence of either of these two signals T cells do not proliferate. It was shown that an apparently diminished recruitment of Lck to the TCR coupled with constitutively active Fyn may result in the delivery of only partial signals to downstream events in the T-cell activation pathway, and may lead to the observed proliferative hyporesponsiveness of anergic T cells $[82,83]$. In this context it is of note that, in contrast to ageing and other autoimmune diseases, T cells from patients with SLE display TCR-mediated signalling alterations associated with defective TCR zeta chain expression [84] reconstitution, which restores IL-2 production [85]. The maintenance of the anergic state is quite well characterized, while the pathways inducing anergy are largely unknown. In the maintenance of anergy in primary T cells, impaired Ras activation seems to play a pivotal role, which is in turn part of the defective TCR-mediated signalling along the PKC-Ras-MAPK pathways [86]. This correlates closely with the reduced activation and activity of ERK2 MAPK that is required for progression to the $S$ phase of the cell cycle, thus explaining altered IL-2 secretion and proliferative hyporesponsiveness.

It is of interest that all these alterations were found with ageing in naïve Tcells as well as in memory T cells $[11,14]$, resulting in proliferative hyporesponsiveness caused, in part, by an alteration of signal transduction pathways, already described in detail. The pivotal PKCRas-MAPK pathway, playing a role in anergy, is also severely impaired with ageing. Moreover, the alteration of the targeting of signalling molecules to the membrane with ageing was also demonstrated for other molecules such as PKC [64]. Recent results indicate that there is a decrease in Lck expression in T-cell lipid rafts in patients with SLE [36] and consequent alterations in intracellular calcium mobilization and the tyrosine phosphorylation pattern of cytosolic proteins. However, this could be inde- pendent of TCR activation. Furthermore, the alteration in the membrane composition and fluidity due to increased cholesterol content could provide an explanation for these signalling alterations $[42,49]$.

The maintenance of anergy thus occurs through an altered TCR signalling, mainly via PKC-Ras-MAPK pathways. This anergy paradoxically may account for the breakdown of the tolerance as seen in autoimmune diseases such as RA as well as during the ageing process. In this scenario, anergy of regulatory T cells, mainly of Th2 cells mediating protection from autoimmune diseases [81], is postulated. The hallmarks of this altered TCR activation upon ligation are the increased levels of basal PTK activity, which correlate with the failure to upregulate PTK activity including Lck and ZAP70, and a decrease in the induction calcium mobilization (extracellular and intracellular) [86], suggesting that the level of Src family tyrosine kinase activity is an important determinant of immune tolerance (reviewed in [87]). This altered signalling leads to a decrease in IL-2 and IL-4 production [88] with concomitant proliferative hyporesponsiveness. It is then clear that cytokines play a major role in regulating immune cells contributing to host defence as well as to autoimmune diseases.

It is also well established that unregulated or changed immune responses driven by cytokines contribute to the pathogenesis of autoimmune diseases. For example, IL-6 knockout mice are totally resistant to collagen-induced arthritis. In this connection, it is known that ageing is associated with high IL-6 levels. Hence, the more we age, the more we become sensitive to a variety of autoimmune-type diseases. In addition to IL-6, other cytokine profile changes occur with ageing. IL-2 is essential for T-cell proliferation, and knocking out this cytokine and its various receptor chains have been associated with the occurrence of autoimmune diseases in mice. It is of note that in ageing, even if it is not associated with a complete lack of IL-2 production (IL-2 'knockout'), the production of this cytokine is commonly severely impaired. It could be then suggested that autoimmune disease may be the clinical manifestation of immunological dysregulation.

Moreover, IL-10 production has been shown to increase with ageing, being part of the well-known Th1/Th2 imbalance in favour of Th2 anti-inflammatory cytokines $[2,6]$. This could be one explanation for the increased incidence of certain diseases with ageing and also, in part, an explanation for many autoimmune diseases. However, this needs further investigation. In fact, it has been demonstrated that IL-10 derived from $\mathrm{CD}^{+} \mathrm{T}$ lymphocytes was increased compared with IL-2 within the rheumatoid synovial membrane $[89,90]$. Hence, excessive IL-10 production occurring with ageing may be involved in the development of arthritis. It could appear paradoxical that an anti-inflammatory cytokine like IL-10 can participate in 
the development of arthritis, but in ageing and in autoimmune diseases there is a complete disequilibrium between proinflammatory cytokines and anti-inflammatory cytokines $[2,6,90]$. In fact, this altered cytokine balance can explain that the increase of anti-inflammatory cytokines suppresses the specific immune response while it favours the development of autoimmune phenomena. Whether IL-10 plays a role in the induction of autoimmune disease is still unknown; we can, however, at least assume that it plays a role in the maintenance of autoimmune diseases.

As mentioned earlier, cytokines are involved in the development of autoimmune diseases. A family of molecules involved in the inhibition of signalling has recently been described [91], namely the suppressors of cytokine signalling. Whether these molecules (SOCS1-SOCS9) are differentially expressed or whether a polymorphisms exists with ageing is unknown, and further studies should be carried out to determine the exact role of the suppressors of cytokine signalling family in autoimmune disorders. Once again, these alterations are not only seen in autoimmune diseases, such as diabetes type 1, SLE and RA [88,92-94], but also in physiological ageing [11]. From this point of view, autoimmune diseases could thus be considered a model of premature ageing (see Fig. 2).

Reciprocally, the decreased activation through the TCR-CD3 complex and CD28-mediated costimulation may render $\mathrm{T}$ cells from individuals with autoimmune disease resistant to the induction of tolerance and activation-induced cell death (apoptosis) [95]. The diminished ability of TCR stimulation to tolerate or eliminate autoreactive T cells, which would normally be inactivated, may thus lead to T-cell escape from negative selection by apoptosis or functional anergy. It was also shown that the second signal may be altered in autoimmune diseases as it is in ageing. Impaired B7 expression on APCs and defects in CD28/B7 costimulation also underlie abnormal T-cell activation in autoimmune diseases [96]. CD28 and a second similar receptor, cytotoxic T leukocyte antigen-4, are positive and negative regulators of T-cell activation, respectively. Cytotoxic Tleukocyte antigen-4 is critical for the induction of peripheral T-cell tolerance and for the deletion of autoreactive T cells [97]. This family of costimulatory molecules is ever expanding [98] and it was very recently demonstrated that the inducible costimulator, the third member of the CD28 family, was directly linked to arthritis. Firstly, inducible costimulator-deficient mice exhibited impaired immunoglobulin class-switching. Moreover, they were very sensitive to experimental autoimmune encephalomyelitis. Finally, inducible costimulator knockout mice were completely resistant to RA [99].

A defect in costimulation may render T cells refractory to TCR-mediated apoptosis or to tolerance-inducing signals. thus result from the fact that the threshold required for TCR activation is markedly increased for T cells in autoimmune diseases. This leads to the already described decreased signalling by PTK in autoimmune diseases [81]. Moreover, the decreased recruitment of ZAP-70 to membrane-bound TCR $\zeta$ could also explain the decreased susceptibility to apoptosis, because this intact association is necessary for the upregulation of FasL on activated T cells and consequently to AICD [100]. Thus, autoreactive T cells that are unable to recruit and activate ZAP-70 cannot upregulate the FasL or cannot ultimately undergo apoptosis via $\mathrm{Fas} / \mathrm{FasL}$ interactions.

Studies suggest that TCR signalling abnormalities are not restricted only to defective apoptosis, but that a cross-talk exists between TCR and Fas for effective T-cell functions [101]. Moreover, in autoimmune lymphoproliferative syndrome many patients display a dysregulated cytokine pattern with dysfunctional Tcells, suggesting that Fas defects due to mutations may impact on pathways of T-cell activation/differentiation. Goldman and colleagues [102] reported the failure of $\mathrm{CD} 4^{+} \mathrm{T}$ cells from autoimmune lymphoproliferative syndrome patients to upregulate surface activation markers including CD25 and CD69, or to produce IFN- $\gamma$ and IL-2 after CD3 stimulation. It is thus suggested that a link exists between the Fas and TCR signalling pathways. The link could be via MAPK pathways, especially JN kinase [103]. This alteration in the cross-talk between the Fas receptor and the TCR via aberrant cytokine secretion may contribute to the development and clinical manifestations of autoimmune diseases. The role of these alterations in ageing and in other autoimmune disorders is still unknown and awaits investigation. However, this could lead to improved understanding of the complex relationships between regulation of signalling pathways and T-cell function (see Fig. 2).

It has recently been shown that activation of the MAPK pathway by T lymphocytes resulted in production of matrix metalloproteinase (MMP-13) by osteoclasts in the context of RA. Moreover, proinflammatory cytokines, including IL-1 and IL-17, synergize to induce the production of MMP-13 via activation of AP-1 (activating protein-1), explaining in part bone loss and cartilage damage [104]. This bone resorption in RA relates to the discovery of osteoclastmediated bone resorption that is regulated by receptor activator of NF-KB ligand. This receptor is also present on $T$ cells and contributes under proinflammatory cytokine stimulation to osteoclast maturation. Altered T-cell signalling thus leads to altered cytokine production, contributing to the pathological hallmarks of RA in the joints (reviewed in [105]). Furthermore, ageing is associated with an increase of oxidative stress, explaining the increased susceptibility to atherosclerosis and cardiovascular diseases. Moreover, it has been proposed that oxidative stress also alters proteins, and this seems to be 


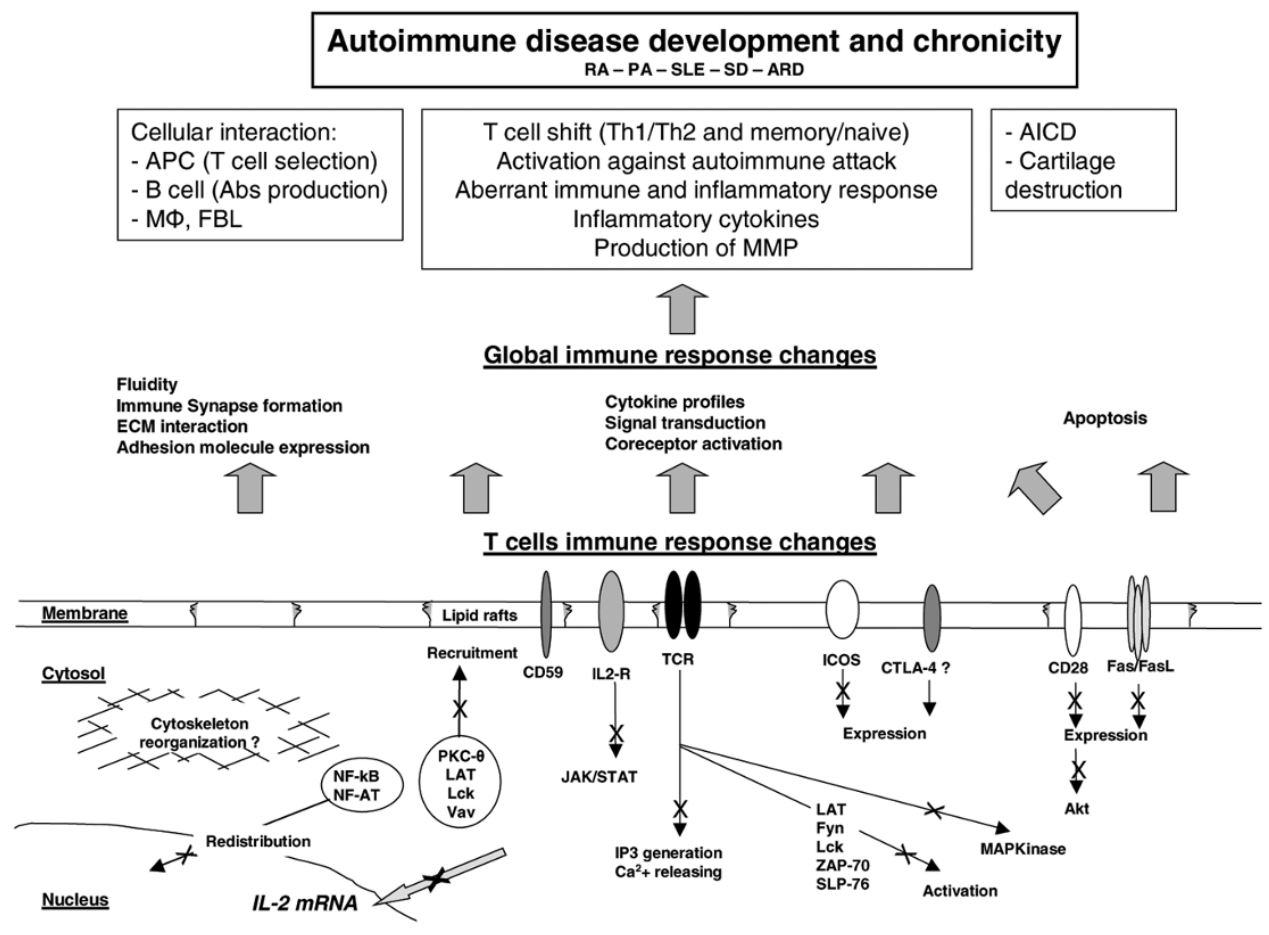

T-lymphocyte effector functions and signalling defects with ageing, role in autoimmune diseases. Defects in many activation pathways and molecules lead to differential effects on T-cell behaviour, and thus on immune responses. Membrane reorganization, cytokine profiles, cellular interactions, and T-cell subpopulation shifts are the consequences of the changes observed in T-cell signalling with ageing. The final consequence is an increased susceptibility to disease that is accompanied by the development of a local environment of a sustained inflammatory state, critical factors for the initiation, the development and the chronicity of autoimmune diseases. Abs, antibodies; AICD, activation-induced cell death; APC, antigen-presenting cell; ARD, autoimmune renal disease; CTLA-4, cytotoxic T-leukocyte antigen-4; ECM, extracellular matrix; FBL, fibroblast; ICOS, inducible costimulatory protein; IL-2R, IL-2 receptor; IP3, myo-inositol 1,4,5-trisphosphate; LAT, linker of activated T cells; MФ, macrophage; MAP, mitogen-activated protein; MMP, matrix metalloproteinase; PA, psoriasis arthritis; PKC, protein kinase C; RA, rheumatoid arthritis; SD, scleroderma; SLE, systemic lupus erythematosus; TCR, T-cell receptor.

another explanation for decreased signalling molecule activation and activity. A role of protein oxidation in the initiation and/or progression of several diseases is indicated by higher levels of oxidized proteins observed in tissues of subjects with diseases such as Alzheimer's disease, Werner's syndrome, and also RA [106]. Hence, the consequences of oxidative stress observed with ageing are also observed in diseases such as RA, contributing to TCR signal transduction changes.

In summary, the alterations found in TCR signalling leading to profound T-cell function changes with ageing, including hyporesponsiveness, decreased tolerance and proneness to apoptosis, may already be found in the early phases of the development of autoimmune diseases such RA. Reciprocally, the alterations render aged individuals more susceptible to autoimmune diseases (see Fig. 3).

\section{Conclusion}

T-cell hyporesponsiveness due to defects of signalling through the TCR either from healthy elderly subjects or from individuals with autoimmune diseases such as RA and SLE results in an impaired ability to mount efficient immune responses and to maintain responsiveness to foreign antigens. This implies that a high proportion of autoreactive T cells might accumulate either intrathymically or in the periphery. T-cell anergy and differential TCR signalling could thus also be key players in the disruption of tolerance and the onset of autoimmune diseases.

Although these studies are still in their infancy, data so far suggest that alterations in TCR signalling and its regulation can significantly contribute to the development of autoimmune diseases. The increasing size of the elderly population may lead to increases in clinically important autoimmune diseases. It is thus imperative to better understand the impact of TCR signalling changes with age on susceptibility to autoimmune disease. This is the only way to develop efficacious prevention and treatment for these diseases. In this respect, further studies on the regulation of cholesterol metabolism in aged T cells may help to understand these signalling alterations and to better 
Figure 3

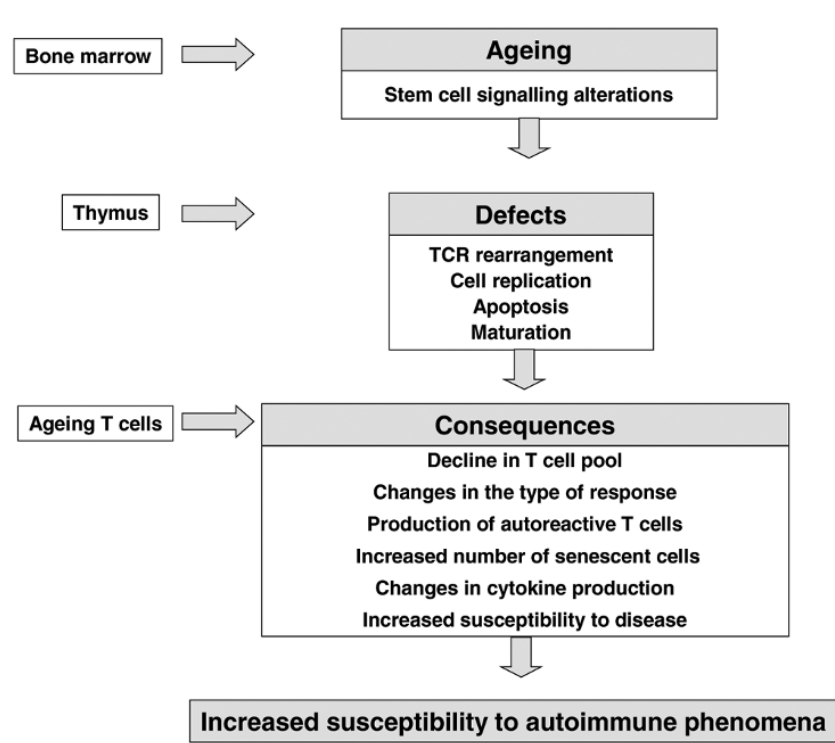

Links between altered T-cell signalling with ageing and susceptibility to autoimmune disease. TCR, T-cell receptor.

define the role of DRMs in ageing and age-related diseases. In addition, studies on T-cell subsets will help to determine whether their responsiveness is selectively affected in aged humans. Current investigations in our laboratories are addressing these questions.

\section{Competing interests}

\section{None declared.}

\section{Acknowledgements}

This work was supported by a grant-in-aid from the National Science and Engineering Research Council of Canada (No. 249549), the Research Center of Ageing of Sherbrooke, the ImAginE consortium and the Canadian Institute of Health Research.

\section{References}

1. Makinodan T, Kay MMB: Age influence on the immune system. Adv Immunol 1980, 29:287-300.

2. Grubeck-Loebenstein B, Wick G: The ageing of the immune system. Adv Immuno/ 2002, 80:243-284.

3. Fulop T Jr, Foris G, Worum I, Leovey A: Age-dependent variations of intralysosomal enzyme release from human PMN leukocytes under various stimuli. Immunobiology 1986, 171: 302-310.

4. Ershler WB: The influence of an ageing immune system on cancer incidence and progression. J Gerontol 1993, 48:B3-B7.

5. Ben-Yehuda A, Weksler ME: Host resistance and the immune system. Clin Ger Med 1992, 8:701-711.

6. Bruunsgaard H, Pedersen AN, Schroll M, Skinhoj P, Pedersen BK: Proliferative responses of blood mononuclear cells (BMNC) in a cohort of elderly humans: role of lymphocyte phenotype and cytokine production. Clin Exp Immunol 2000, 119:433-440.

7. Effros RB: Costimulatory mechanisms in the elderly. Vaccine 2000, 18:1661-1665.

8. Liparoto SF, Myszka DG, Wu Z, Goldstein B, Laue TM, Ciardelli $T L$ : Analysis of the role of the interleukin-2 receptor gamma chain in ligand binding. Biochemistry 2002, 41:2543-2551.
9. Hombach A, Sent D, Schneider C, Heuser C, Koch D, Pohl C, Seliger B, Abken H: T-cell activation by recombinant receptors: CD28 costimulation is required for interleukin 2 secretion and receptor-mediated T-cell proliferation but does not affect receptor-mediated target cell lysis. Cancer Res 2001, 61: 1976-1982.

10. Nagel JE, Chopra RK, Dowers DC, Adler WH: Effect of age on the human high affinity interleukin 2 receptor of phytohaemagglutinin stimulated peripheral blood lymphocytes. Clin Exp Immunol 1989, 75:286-291.

11. Pawelec G, Hirokawa K, Fülöp T: Altered T cell signalling in ageing. Mech Ageing Dev 2001, 122:1613-1657.

12. Fulop T Jr: Signal transduction changes in granulocytes and lymphocytes with ageing. Immunol Lett 1994, 40:259-268.

13. Ghosh J, Miller RA: Rapid tyrosine phosphorylation of Grb2 and Shc in T cells exposed to anti-CD3, anti-CD4, and antiCD45 stimuli: differential effects of ageing. Mech Ageing Dev 1995, 80:171-187.

14. Miller RA: Effect of ageing on T lymphocyte activation. Vaccine 2000, 18:1654-1660

15. Fulop T Jr, Gagné D, Goulet AC, Desgeroges S, Lacombe G, Arcand M, Dupuis G: Age-related impairment of p56lck and ZAP70 activities in human T lymphocytes activated through the TCR/CD3 complex. Exp Gerontol 1999, 34:197-216.

16. Margulies DH: Interactions of TCRs with MHC-peptide complexes - a quantitative basis for mechanistic models. Curr Opin Immunol 1997, 9:390-395.

17. Hermiston ML, Xu Z, Majeti R, Weiss A: Reciprocal regulation of lymphocyte activation by tyrosine kinases and phosphatases. $J$ Clin Invest 2002, 109:9-14.

18. Nel AE: T-cell activation through the antigen receptor. Part 1: signaling components, signaling pathways, and signal integration at the T-cell antigen receptor synapse. J Allergy Clin Immunol 2002, 109:758-770.

19. Nel AE, Slaughter N: T-cell activation through the antigen receptor. Part 2: role of signaling cascades in T-cell differentiation, anergy, immune senescence, and development of immunotherapy. J Allergy Clin Immunol 2002, 109:901-915.

20. Billadeau DD, Leibson PJ: ITAMs versus ITIMs: striking a balance during cell regulation. J Clin Invest 2002, 109:161-168.

21. Walk SF, March ME, Ravichandran KS: Roles of Lck, Syk and ZAP-70 tyrosine kinases in TCR-mediated phosphorylation of the adapter protein Shc. Eur J Immunol 1998, 28:2265-2275.

22. Sasaki T, Sasaki-Irie J, Penninger JM: New insights into the transmembrane protein tyrosine phosphatase CD45. Intern J Biochem Cell Biol 2001, 11:1041-1046.

23. Bosselut R, Zhang WG, Ashe JM, Kopacz JL, Samelson LE, Singer A: Association of the adaptor molecule LAT with CD4 and CD8 coreceptors identifies a new coreceptor function in T cell receptor signal transduction. J Exp Med 1999, 190:15171526.

24. Kane LP, Lin J, Weiss A: Signal transduction by the TCR for antigen. Curr Opin Immunol 2000, 12:242-249.

25. Wulfing C, Rabinowitz JD, Beeson C, Sjaastad MD, Mcconnell HM, Davis MM: Kinetics and extent of T cell activation as measured with the calcium signal. J Exp Med 1997, 185:18151825.

26. Dong C, Davis RJ, Flavell RA: MAP kinases in the immune response. Annu Rev Immunol 2002, 20:55-72.

27. Guse $\mathrm{AH}: \mathrm{Ca}^{2+}$ signaling in T-lymphocytes. Crit Rev Immunol 1998, 18:419-448.

28. Bi K, Tanaka Y, Coudronniere N, Sugie K, Hong S, van Stipdonk MJB, Altman A: Antigen-induced translocation of PKC- $\theta$ to membrane rafts is required for $\mathrm{T}$ cell activation. Nat Immunol 2001, 2:556-563.

29. Penninger JM, Crabtree GR: The actin cytoskeleton and lymphocyte activation. Cell 1999, 96:9-12.

30. Wells AD, Gudmundsdottir H, Turka LA: Following the fate of individuals $\mathrm{T}$ cells throughout activation and clonal expansion - signals from T cell receptor and CD28 differentially regulate the induction and duration of a proliferative reponse. J Clin Invest 1997, 100:3173-3183.

31. Frauwirth KA, Thompson CB: Activation and inhibition of lymphocytes by costimulation. J Clin Invest 2002, 109:295-299.

32. Dustin ML: Membrane domains and the immunological synapse: keeping T cells resting and ready. J Clin Invest 2002, 109:155-160. 
33. Brown DA, London E: Functions of lipid rafts in biological membranes. Annu Rev Cell Dev Biol 1998, 14:111-136.

34. Zhang W, Trible RP, Samelson LE: LAT palmitoylation: its essential role in membrane microdomain targeting and tyrosine phosphorylation during T cell activation. Immunity 1998, 9:239246.

35. Janes PW, Ley SC, Magee Al, Kabouridis PS: The role of lipid rafts in T cell antigen receptor (TCR) signalling. Semin Immunol 2000, 12:23-34.

36. Jury EC, Kabouridis PS, Abba A, Mageed RA, Isenberg DA: Increased ubiquination and reduced expression of Lck in $T$ lymphocytes from patients with systemic lupus erythematosus. Arthritis Rheumatism 2003, 48:1343-1354.

37. Lio D, Candore G, Cigna D, Danna C, Dilorenzo G, Giordano C Lucania G, Mansueto P, Melluso M, Modica MA, Caruso C: In vitro $T$ cell activation in elderly individuals: failure in CD69 and CD71 expression. Mech Ageing Dev 1996, 89:51-58.

38. Tamura T, Kunimatsu T, Yee ST, Igarashi O, Utsuyama M, Tanaka $\mathrm{S}$, Miyazaki S, Hirokawa K, Nariuchi H: Molecular mechanism of the impairment in activation signal transduction in CD4(+) $\mathrm{T}$ cells from old mice. Int Immuno/ 2000, 12:1205-1215.

39. Boucher N, Dufeu-Duchesne T, Vicaut E, Farge D, Effros RB, Schächter F: CD28 expression in T cell ageing and human longevity. Exp Gerontol 1998, 33:267-282.

40. Simons K, Ikonen E: Functional rafts in cell membranes. Nature 1997, 387:569-572.

41. Von Haller PD, Donohoe S, Goodlett DR, Aebersold R, Watts JD: Mass spectrometric characterization of proteins extracted from Jurkat T cell detergent-resistant membrane domains. Proteomics 2001, 1:1010-1021.

42. Zs-Nagy I, Kitani K, Ohta M, Zs-Nagy V, Imahori K: Age-dependent decrease of the lateral diffusion constant of proteins in the plasma membrane of hepatocytes as revealed by fluorescent recovery after photobleaching in tissue smears. Arch Gerontol Geriatr 1986, 5:131-146.

43. Silvius JR: Role of cholesterol in lipid raft formation: lessons from lipid model systems. Biochem Biophys Acta 2003, 1610: 174-183.

44. Eisenbraun MD, Tamir A, Miller RA: Altered composition of the immunological synapse in anergic, age-dependent memory T cell subset. J Immuno/ 2000, 164:6105-6112.

45. Garcia GG, Miller RA: Single-cell analyses reveal two defects in peptide-specific activation of naive T cells from aged mice. J Immunol 2001, 166:3151-3157.

46. Marmor $M D$, Julius $M$ : Role of lipid rafts in regulating interleukin-2 receptor signaling. Blood 2001, 98:1489-1497.

47. Fülöp $T \mathrm{Jr}$, Douziech $N$, Goulet AC, Desgeorges $S$, Linteau $A$ Lacombe G, Dupuis G: Cyclodextrin modulation of T lymphocyte signal transduction with ageing. Mech Age Dev 2001, 152: 1413-1430.

48. Incardona JP, Eaton S: Cholesterol in signal transduction. Curr Opin Cell Biol 2000, 12:193-203.

49. Rivney B, Bergman S, Shinitzky M, Globerson A: Correlation between membrane viscosity, serum cholesterol, lymphocyte activation and ageing in men. Mech Age Dev 1980, 12:119-126.

50. Douziech N, Seres I, Larbi A, Szikszay E, Roy PM, Arcand A, Dupuis D, Fülöp T Jr: Modulation of human lymphocyte proliferative response with ageing. Exp Gerontol 2002, 37:369-377.

51. Kitani K: Lateral mobility of proteins and lipids of cell surface membranes during ageing: do the data support 'The membrane hypothesis of ageing'? Mech Ageing Dev 1999, 107:299322.

52. Viola A, Schroeder S, Sakakibara Y, Lanzavecchia A: T lymphocyte costimulation mediated by reorganization of membrane microdomains. Science 1999, 283:680-682.

53. Janes PW, Ley SC, Magee Al: Aggregation of lipid rafts accompanies signaling via the $\mathrm{T}$ cell antiger receptor. J Cell Biol 1999, 147:447-461.

54. Miller RA, Garcia G, Kirk CJ, Witkowski JM: Early activation defects in T lymphocytes from aged mice. Immunol Rev 1997, 150:79-90

55. Fülöp $T \mathrm{Jr}$, Douziech N, Larbi A, Dupuis G: The role of lipid rafts in T lymphocyte signal transduction with ageing. Ann NY Acad Sci 2002, 973:302-304.

56. Koretzky GA, Myung PS: Positive and negative regulation of Tcell activation by adaptor proteins. Nat Rev Immunol 2001, 1:95-107.
57. Franceschi $\mathrm{C}$, Bonafe $\mathrm{M}$, Valensin $\mathrm{S}$, Olivieri $\mathrm{F}$, De Luca $\mathrm{M}$, Ottaviani E, De Benedictis G: Inflamm-ageing. An evolutionary perspective on immunosenescence. Ann NY Acad Sci 2000, 908: 244-254

58. Kabouridis PS, Janzen J, Magee AL, Ley SG: Cholesterol depletion disrupts lipid rafts and modulates the activity of multiple signaling pathways in T lymphocytes. Eur J Immunol 2000, 30: 954-963.

59. Harman D: Ageing: a theory based on free radical and radiation chemistry. J Gerontol 1956, 11:298-300.

60. Pahlavani MA, Harris MD: Effect of in vitro generation of oxygen free radicals on $\mathrm{T}$ cell function in young and old rats. Free Radic Biol Med 1997, 25:903-913.

61. Whisler RL, Karanfilov Cl, Newhouse YG, Fox CC, Lakshmanan RR, Liu BQ: Phosphorylation and coupling of zeta-chains to activated T-cell receptor (TCR)/CD3 complexes from peripheral blood T-cells of elderly humans. Mech Age Dev 1998 105:115-135.

62. Garcia GG, Miller RA: Increased Zap-70 association with CD3zeta in CD4 T cells from old mice. Cell Immunol 1998 190:91-100

63. Quadri RA, Plastre O, Phelouzat MA, Arbogast A., Proust JJ: Agerelated tyrosine-specific protein phosphorylation defect in human $\mathrm{T}$ lymphocytes activated through CD3, CD4, CD8 or the IL-2 receptor. Mech Ageing Dev 1996, 88:125-138.

64. Miller RA, Jacobson B, Weil G, Simons ERJ: Diminished calcium influx in lectin-stimulated T cells from old mice. J Cell Physiol 1987, 132:337-342.

65. Proust JJ, Fiburn CR, Harrison SA, Buchholz MA, Nordin AA: Agerelated defect in signal transduction during lectin activation of murine T lymphocytes. J Immunol 1987, 139:1472-1478.

66. Whisler RL, Bagenstose SE, Newhouse YG, Carle KW: Expression and catalytic activites of protein tyrosine kinases (PTKs) Fyn and Lck in peripheral blood T cells from elderly humans stimulated through the T cell receptor (TCR)/CD3 complex. Mech Ageing Dev 1997, 98:57-73.

67. Li M, Walter R, Torres C, Sierra F: Impaired signal transduction in mitogen activated rat splenic lymphocytes during ageing Mech Age Dev 2000, 113:85-99.

68. Whisler RL, Newhouse YG, Bagenstose SE: Age-related reductions in the activation of mitogen-activated protein kinases p44mapk/ERK1 and p42mapk/ERK2 in human T cells stimulated via ligation of the T cell receptor complex. Cell Immunol 1996, 168:201-210.

69. Hutter D, Yo Y, Chen W, Liu P, Holbrook NJ, Roth GS, Liu Y: Age-related decline in Ras/ERK mitogen-activated protein kinase cascade is linked to a reduced association between Shc and EGF receptor. J Geronto/ 2000, 55A:B125-B134.

70. Pahlavani MA, Harris MD, Richardson A: Activation of p21(ras)/MAPK signal transduction molecules decreases with age in mitogen-stimulated T cells from rats. Cell Immunol 1995, 165:84-91.

71. Ponnappan U, Trebilcock GU, Zheng MZ: Studies into the effect of tyrosine phosphatase inhibitor phenylarsine oxide on NFkappaB activation in T lymphocytes during ageing: evidence for altered IkappaB-alpha phosphorylation and degradation. Exp Gerontol 1999, 34:95-107.

72. Lanzavecchia A, Sallusto F: Dynamics of $T$ lymphocyte responses intermediates, effectors and memeory cells. Science 2000, 290:92-97.

73. Fagnoni FF, Vescovini R, Passeri G, Bologna G, Pedrazzoni M, Lavagetto G, Casti A, Franceschi C, Passeri M, Sansoni P: Shortage of circulating naive $\mathrm{CD} 8(+) \mathrm{T}$ cells provides new insights on immunodeficiency in ageing. Blood 2000, 95:2860-2868.

74. Pawelec G, Adibzadeh M, Solana R, Beckman I: The T cell in the ageing individual. Mech Ageing Dev 1997, 93:35-45.

75. Kukreja A, Cost G, Marker J, Zhang C, Sun Z, Lin-Su K, Ten S, Sanz M, Exley M, Wilson B, Porcelli S, Maclaren N: Multiple immuno-regulatory defects in type-1 diabetes. J Clin Invest 2002, 109:131-140.

76. Gorozny JJ, Weyand CM: Thymic function and peripheral T cell homeostasis in rheumatoid arthritis. TRENDS Immunol 2001 22:251-255.

77. Theofilopoulos AN, Dummer W, Kono DH: T cell homeostasis and systemic autoimmunity. J Clin Invest 2001, 108:335-340.

78. Freitas AA, Rocha B: Population biology of lymphocytes: the flight for survival. Annu Rev Immunol 2000, 18:83-111. 
79. Ernst B, Lee DS, Chang JM, Sprent J, Surh CD: The peptide ligands mediating positive selection in the thymus control $T$ cell survival and homeostatic proliferation in the periphery. Immunity 1999, 11:173-181.

80. Goldrath AW, Bevan MJ: Selecting and maintaining a divers $T$ cell repertoire. Nature 1999, 402:255-262.

81. Jendro MC, Ganten T, Matteson EL, Weyand CM, Goronzy JJ: Emergence of oligoclonal $T$ cell populations following therapeutic $\mathrm{T}$ cell depletion in rheumatoid arthritis. Arthritis Rheum 1995, 38:1242-1251.

82. Salojin KV, Zhang J, Madrenas J, Delovitch TL: T-cell anergy and altered T-cell receptor signalling: effects on autoimmune disease. Immunol Today 1998, 19:468-473.

83. Zipris $D$, Lazarus AH, Crow AR, Hadzija M, Delovitch TL: Defective thymic $T$ cell activation by concanavalin A and anti-CD3 in autoimmune nonobese diabetic mice. Evidence for thymic $T$ cell anergy that correlates with the onset of insulitis. $J$ Immunol 1991, 146:3763-3771.

84. Nambiar MP, Mitchell JP, Cerutti RP, Malloy MA, Tsokos GC: Prevalence of $\mathrm{T}$ cell receptor zeta chain deficiency in systemic lupus erythematosus. Lupus 2003, 12:46-51.

85. Nambiar MP, Fisher CU, Warke WG, Krishnan S, Mitchell JP, Delaney N, Tsokos GC: Reconstitution of deficient T cell receptor zeta chain restores $T$ cell signalling and augments $T$ cell receptor/CD3-induced interleukine 2 production in patients systemic lupus erythematosus. Arthritis Rheum 2003, 48: 1948-1955.

86. Gajewski TF, Quian D, Fields P, Fitch FW: Anergic T-lymphocyte clones have altered inositol phosphate, calcium, and tyrosine kinase signaling pathways. Proc Natl Acad Sci USA 1994, 91: 38-42.

87. Yu CC, Mamchak AA, DeFranco AL: Signaling mutations and autoimmunity. Curr Dir Autoimmun 2003, 6:61-88.

88. Rapaport MJ, Lazarus AH, Jaramillo A, Speck E, Delovitch TL: Thymic $T$ cell anergy in autoimmune nonobese diabetic mice is mediated by deficient $\mathrm{T}$ cell receptor regulation of the pathway of p21ras activation. J Exp Med 1993, 177:12211226.

89. Cohen SB, Katsikis PD, Thomssen $H$, Webb LM, Maini RN, Londei M, Feldmann M: High level of interleukin-10 production by the activated $T$ cell population within the rheumatoid synovial membrane. Arthritis Rheum 1995, 38:946-952.

90. Moller B, Nguyen TT, Kessler U, Kaltwasser JP, Hoelzer D, Ottmann OG: Interleukin-10 expression: is there a neglected contribution of CD8+ T cells in rheumatoid arthritis joints? Clin Exp Rheumatol 2002, 20:813-822.

91. Yoshimura A, Ohkubo T, Kiguchi T, Jenkins NA, Gilbert DJ, Copeland NG, Hara T, Miyajima A: A novel cytokine-inducible gene CIS encodes an SH2-containing protein that binds to tyrosine-phosphorylated interleukin 3 and erythropoietin receptors. EMBO J 1995, 14:2816-2826.

92. Rapoport MJ, Jaramillo A, Zipris D, Lazarus AH, Serreze DV, Leiter EH, Cyopick P, Danska JS, Delovitch TL: Interleukin 4 reverses $T$ cell proliferative unresponsiveness and prevents the onset of diabetes in nonobese diabetic mice. J Exp Med 1993, 178: 87-99.

93. Zipris D, Crow AR, Delovitch TL: Altered thymic and peripheral T-lymphocyte repertoire preceding onset of diabetes in NOD mice. Diabetes 1991, 40:429-435.

94. Portales-Perez D, Gonzalez-Amaro R, Abud-Mendoza C, SanchezArmass S: Abnormalities in CD69 expression, cytosolic $\mathrm{pH}$ and $\mathrm{Ca}^{2+}$ during activation of lymphocytes from patients with systemic lupus erythematosus. Lupus 1997, 6:48-56.

95. Piecyk $M$, Anderson $P$ : Signal transduction in rheumatoid arthritis. Best Practice Res Clin Rheumatol 2001, 15:789-803.

96. Zhang J, Bardos T, Mikecz K, Finnegan A, Glant TT: Impaired Fas signalling pathway is invovolved in defective $T$ cell apoptosis in autoimmune murine arthritis. J Immunol 2001, 166:49814986.

97. Garcia-Cozar FJ, Molina IJ, Cuadrado MJ, Marubayashi M, Pena J, Santamaria M: Defective B7 expression on antigen-presenting cells underlying $\mathrm{T}$ cell activation abnormalities in systemic lupus erythematosus (SLE) patients. Clin Exp Immunol 1996, 104:72-79.

98. Van Parijs L, Abbas AK: Homeostasis and self-tolerance in the immune system: turning lymphocytes off. Science 1998, 280: 243-248.
99. Watanabe N, Gavrieli M, Sedy JR, Yang J, Fallarino F, Loftin SK, Hurchla MA, Zimmerman N, Sim J, Zang X, Murphy TL, Russell JH, Allison JP, Murphy KM: BTLA is a lymphocyte inhibitory receptor with similarities to CTLA-4 and PD-1. Nat Immunol 2003, 4:670-679.

100. Nurieva RI, Treuting P, Duong J, Flavell RA, Dong C: Inducible costimulator is essential for collagen-induced arthritis. J Clin Invest 2003, 111:701-706.

101. Sahuquillo AG, Roumier A, Teixeiro E, Bragado R, Alarcon B: T cell receptor (TCR) engagement in apoptosis-defective, but interleukin 2 (IL-2)-producing, T cells results in impaired ZAP70/CD3-zeta association. J Exp Med 1998, 187:1179-1192.

102. Goldman FD, Vighakar R, Puck JM, Straus SE, Ballas ZK, Hollenback C, Loew T, Thompson A, Song K, Cook RT: Aberrant T cell antigen receptor mediated responses in autoimmune lymphoproliferative syndrome. Clin Immuno/ 2002, 104:31-39.

103. Wilson DJ, Fortner KA, Lynch DH: JNK, but not MAPK, activation is associated with Fas mediated apoptosis in T cells. Eur $J$ Immunol 1996, 26:989-994.

104. Rifas L, Arackal S: T cells regulate the expression of matrix metalloproteinase in human osteoblasts via a dual mitogenactivated protein kinase mechanism. Arthritis Rheum 2003, 4: 993-1001.

105. Firestein GS: Evolving concepts of rheumatoid arthritis. Nature 2003, 423:356-361.

106. Chapman ML, Rubin BR, Gracy RW: Increased carbonyl content of protein in synovial fluid from patients with rheumatoid arthritis. J Rheumatol 1989, 16:15-18.

\section{Correspondence}

Dr Tamàs Fülöp Jr, Research Center on Ageing, University of Sherbrooke, 1036 Belvedere Street South, Sherbrooke, Quebec, Canada J1H 4C4. Fax: +1 8198213141 ; e-mail: Tamas.Fulop@USherbrooke.ca 